\title{
Scrub Typhus and Molecular Characterization of Orientia tsutsugamushi from Central Nepal
}

\author{
Rajendra Gautam ${ }^{1}$, Keshab Parajuli ${ }^{1}$, Mythili Tadepalli ${ }^{2}$, Stephen Graves ${ }^{2}$, John Stenos ${ }^{2, *}$ \\ and Jeevan Bahadur Sherchand ${ }^{1}$ \\ 1 Department of Microbiology, Maharajgunj Medical Campus, Institute of Medicine, Kathmandu 44600, Nepal; \\ gautamrajen@gmail.com (R.G.); drkparajuli@iom.edu.np (K.P.); jeevanbsherchand@gmail.com (J.B.S.) \\ 2 Australian Rickettsial Reference Laboratory, Geelong, VIC 3220, Australia; \\ mythili.tadepalli@barwonhealth.org.au (M.T.); graves.rickettsia@gmail.com (S.G.) \\ * Correspondence: john.stenos@barwonhealth.org.au; Tel.: +61-342151357
}

Citation: Gautam, R.; Parajuli, K.; Tadepalli, M.; Graves, S.; Stenos, J.; Sherchand, J.B. Scrub Typhus and Molecular Characterization of Orientia tsutsugamushi from Central Nepal. Pathogens 2021, 10, 422. https://doi.org/10.3390/ pathogens10040422

Academic Editor: Fabrizio Bruschi

Received: 15 March 2021

Accepted: 30 March 2021

Published: 1 April 2021

Publisher's Note: MDPI stays neutral with regard to jurisdictional claims in published maps and institutional affiliations.

Copyright: (c) 2021 by the authors. Licensee MDPI, Basel, Switzerland. This article is an open access article distributed under the terms and conditions of the Creative Commons Attribution (CC BY) license (https:// creativecommons.org/licenses/by/ $4.0 /)$.

\begin{abstract}
Scrub typhus is a vector-borne, acute febrile illness caused by Orientia tsutsugamushi. Scrub typhus continues to be an important but neglected tropical disease in Nepal. Information on this pathogen in Nepal is limited to serological surveys with little information available on molecular methods to detect O. tsutsugamushi. Limited information exists on the genetic diversity of this pathogen. A total of 282 blood samples were obtained from patients with suspected scrub typhus from central Nepal and 84 (30\%) were positive for O. tsutsugamushi by $16 \mathrm{~S}$ rRNA qPCR. Positive samples were further subjected to $56 \mathrm{kDa}$ and $47 \mathrm{kDa}$ molecular typing and molecularly compared to other O. tsutsugamushi strains. Phylogenetic analysis revealed that Nepalese O. tsutsugamushi strains largely cluster together and cluster away from other O. tsutsugamushi strains from Asia and elsewhere. One exception was the sample of Nepal_1, with its partial 56 kDa sequence clustering more closely with non-Nepalese O. tsutsugamushi $56 \mathrm{kDa}$ sequences, potentially indicating that homologous recombination may influence the genetic diversity of strains in this region. Knowledge on the circulating strains in Nepal is important to the development of diagnostic tests and vaccines to support public health measures to control scrub typhus in this country.
\end{abstract}

Keywords: vector; molecular typing; 56 kDa; 47 kDa; qPCR

\section{Introduction}

Scrub typhus is a vector-borne, acute febrile illness caused by Orientia tsutsugamushi and transmitted predominantly by mite larvae ("chiggers") of the genus Leptotrombidium (usually species deliense) [1]. The rapid and accurate diagnosis of rickettsial illness is important for the appropriate antibiotic treatment. Traditionally, the diagnosis of scrub typhus is based on serological tests. Among the various serological tests, indirect immune fluorescence assay (IFA) is the most sensitive and specific test, and considered to be the gold standard test, but it is not usually positive in the acute phase of the disease as antibody levels become positive during convalescence [2]. The most sensitive test for the detection of O. tsutsugamushi is a culture, but it requires a BSL-3 laboratory and can take up to 60 days to yield a positive result, limiting its use in clinical diagnosis of this infectious disease [2]. As an alternative, polymerase chain reaction (PCR) is a highly sensitive and specific molecular detection method that can be used for the direct detection of O. tsutsugamushi DNA in blood and eschar samples [3]. However, it is not widely used in resource-limited settings.

O. tsutsugamushi strains can be differentiated serologically, primarily through immunoassays targeting the $O$. tsutsugamushi $56 \mathrm{kDa}$ type-specific antigen. The $56 \mathrm{kDa}$ type-specific antigen gene encodes a membrane protein, which accounts for $10-15 \%$ of the total protein of O. tsutsugamushi, and is highly immunogenic in humans [4]. This protein [5], along with others, including the O. tsutsugamushi $47 \mathrm{kDa}$ antigen [6], has been 
the target of ongoing efforts to develop scrub typhus vaccines. To support efforts to determine the coverage of vaccines targeting these immunodominant $O$. tsutsugamushi proteins, but also to understand the broader genetic diversity of this intracellular pathogen, the genes encoding these proteins have been extensively studied. Molecular typing studies of the $O$. tsutsugamushi $56 \mathrm{kDa}$ protein gene have revealed significant divergence among $O$. tsutsugamushi isolates of $>80 \%$ nucleotide similarity [7-9]. In contrast, molecular typing of the O. tsutsugamushi $47 \mathrm{kDa}$ protein gene revealed less diversity than that observed for the $56 \mathrm{kDa}$ protein gene, with $96.7 \%$ to $100 \%$ sequence identity reported among a geographically diverse group of strains [6].

Scrub typhus is reported from several geographical areas of Nepal and accounts for $25 \%-40 \%$ of acute febrile illness $[10,11]$. Currently, there is a lack of information on the molecular detection and characterization of the strains of $O$. tsutsugamushi that occurs in Nepal. Hence, this study was conducted to initially detect $O$. tsutsugamushi and to molecularly characterize the strains in order to assist with diagnostic assay development.

\section{Results}

Rickettsial qPCR screening of 282 blood samples obtained from patients with suspected scrub typhus from central Nepal revealed an overall positivity of $29.8 \%(84 / 282)$.

Seventeen positive samples with relatively strong $\mathrm{Ct}$ values between 24 and 32 were subjected to conventional PCR amplification and sequencing of the $56 \mathrm{kDa}$ and $47 \mathrm{kDa}$ genes, obtaining partial sequences for both genes from six samples and a further four $47 \mathrm{kDa}$ sequences from an additional four samples. The 10 samples from which $56 \mathrm{kDa}$ and $47 \mathrm{kDa}$ gene sequences were amplified were named Nepal_1-10.

A BlastN analysis of Nepal 1-10 47kDa sequences revealed that they shared between $89.7 \%$ and $99.3 \%$ sequence similarity to the O. tsutsugamushi $47 \mathrm{kDa}$ sequences deposited in Genbank. A neighbor-joining bootstrap phylogenetic tree was constructed (Figure 1) to gain insight into the phylogenetic relationships of these novel O. tsutsugamushi $47 \mathrm{kDa}$ sequences to those previously described. Neighbor-joining analysis revealed that Nepalese O. tsutsugamushi strains clustered together and clustered away from other strains detected in the Middle East and Asia (Figure 1). The 10 sequences of O. tsutsugamushi $47 \mathrm{kDa}$ were subsequently submitted to Genbank (Genbank Accession Number \#MW657371MW657380).

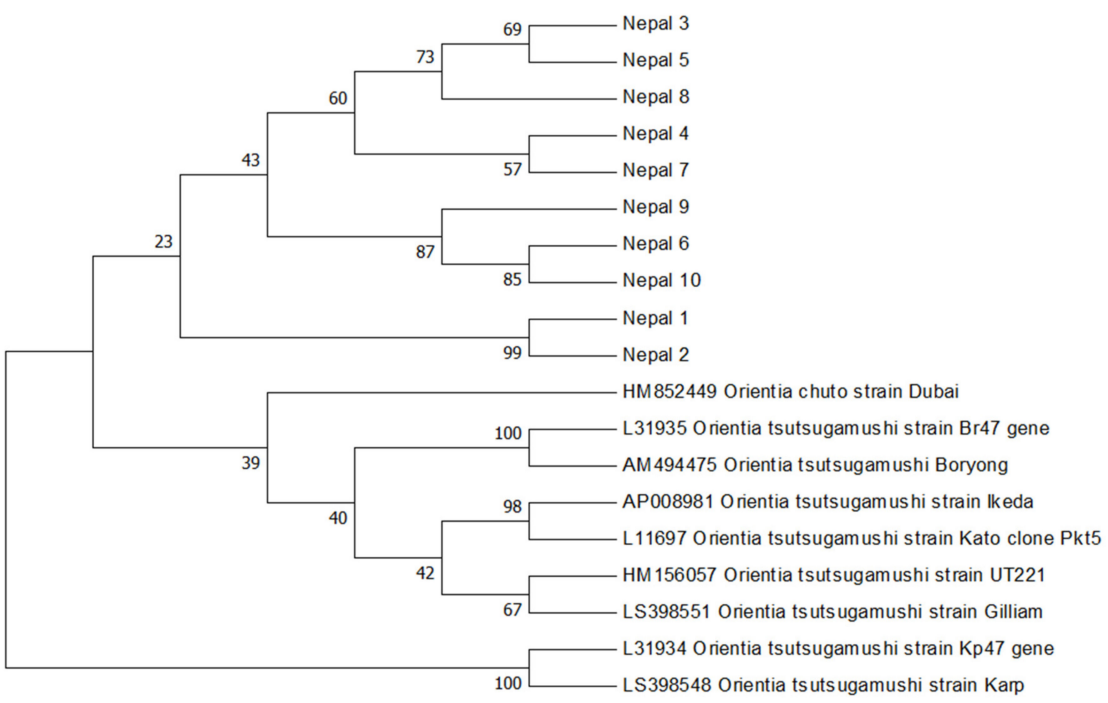

Figure 1. Orientia tsutsugamushi $47 \mathrm{kDa}$ gene bootstrap consensus phylogenetic tree. The phylogenetic tree was inferred using the neighbor-joining method. The percentage of replicate trees in which the associated taxa clustered together in the bootstrap test (1000 replicates) are shown next to the branches. The Genbank accession numbers for the $O$. tsutsugamushi strains retrieved are indicated. 
Further molecular characterization was obtained by sequencing and phylogenetic analysis of the six $56 \mathrm{kDa}$ gene fragments successfully amplified from Nepal_1, Nepal_4-6, and Nepal_9-10. BlastN searching of Genbank confirmed that these partial Nepalese $O$. tsutsugamushi $56 \mathrm{kDa}$ sequences shared between $93.3 \%$ and $99.0 \%$ similarity to O. tsutsugamushi $56 \mathrm{kDa}$ sequences deposited in Genbank. The results of the phylogenetic analysis of the amplified Nepalese $O$. tsutsugamushi $56 \mathrm{kDa}$ sequences were largely consistent with the analysis of the amplified $47 \mathrm{kDa}$ sequences, demonstrating that the Nepalese strains form a phylogenetically distinct clade from $O$. tsutsugamushi strains detected elsewhere (Figure 2). The exception was the Nepal_1 $56 \mathrm{kDa}$ sequence, which was observed forming its own subclade within a phylogenetically distinct clade and away from other Nepalese $O$. tsutsugamushi $56 \mathrm{kDa}$ sequences. The six novel $O$. tsutsugamushi $56 \mathrm{kDa}$ sequences were subsequently submitted to Genbank (Genbank Accession Number \#MW657365-MW657370).

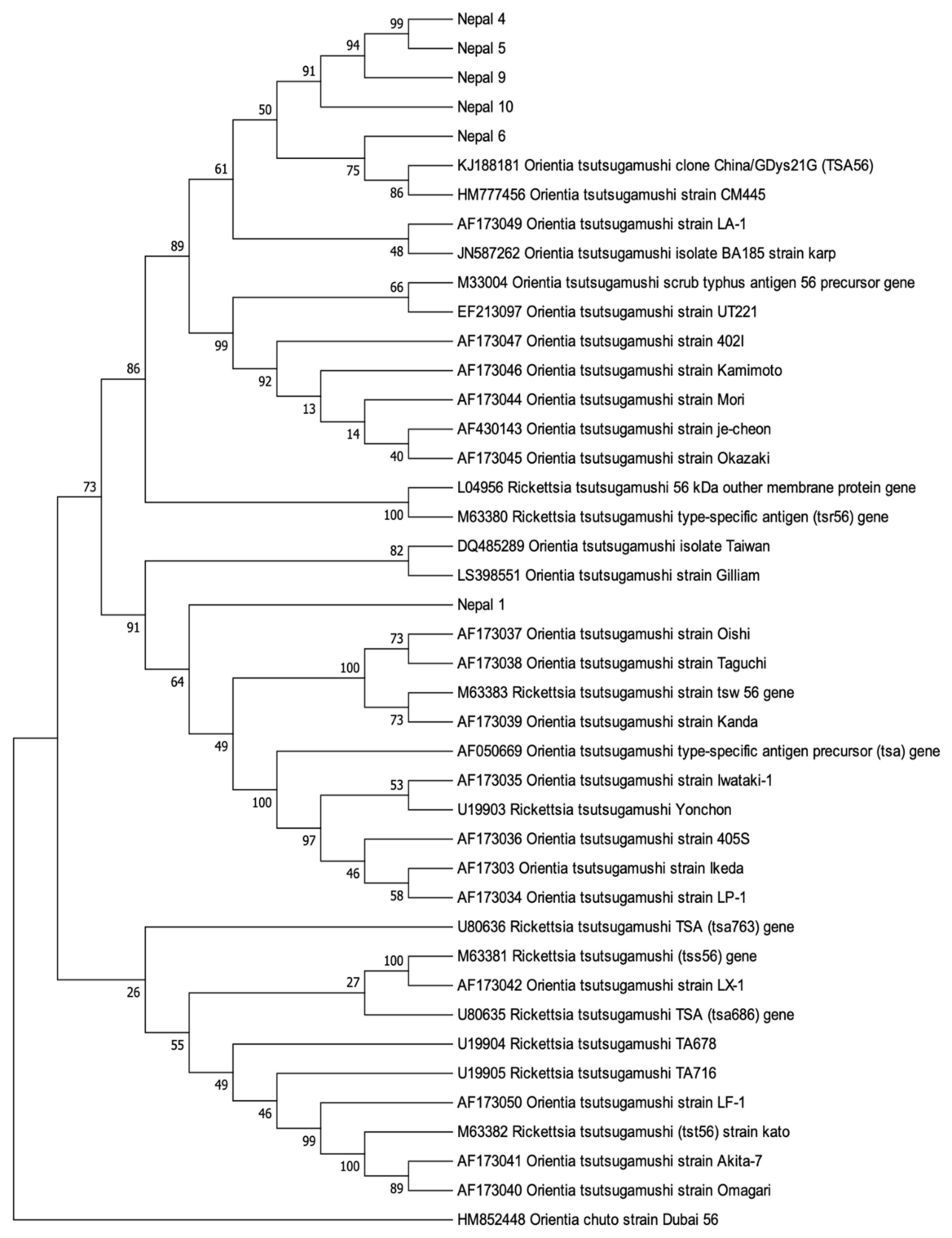

Figure 2. Orientia tsutsugamushi $56 \mathrm{kDa}$ gene bootstrap consensus phylogenetic tree. The phylogenetic tree was inferred using the neighbor-joining method. The percentage of replicate trees in which the associated taxa clustered together in the bootstrap test (1000 replicates) are shown next to the branches. The Genbank accession numbers for the $O$. tsutsugamushi strains retrieved are indicated. 


\section{Discussion}

Scrub typhus is emerging as a major public health problem in Nepal with reports of its occurrence from several widely reported geographic areas $[12,13]$. Despite reports of serological evidence of scrub typhus [10], Nepalese molecular surveys have been largely limited to studies of chiggers mite samples and samples from rodents [12]. To date, no molecular studies have been performed to characterize O. tsutsugamushi, the causative agent of scrub typhus, from Nepal.

In the current study, we used PCR to screen for the presence of O. tsutsugamushi, in blood samples from people with suspected scrub typhus. A prevalence of $29.8 \%$ was detected in these samples, confirming the presence of O. tsutsugamushi and the suspected diagnosis of scrub typhus in almost a third of these patients. This result is similar to the results of a previous serological survey of blood samples collected from Nepalese patients with acute febrile illness, where $40.3 \%$ of blood samples were positive for IgM antibodies against $O$. tsutsugamushi [10]. Notably, the positive patients from this study originated from the Chitwan district in central Nepal. This and adjacent localities were previously shown to be scrub typhus "hotspots" in Nepal, with the Chitwan district itself responsible for $34.4 \%$ of the 831 cases of this vector-borne disease in 2016 [12].

Molecular methods such as PCR are the most useful for the diagnosis of scrub typhus in the early stage of disease, before the antibodies are detectable by serological methods. It was demonstrated previously that the performance of PCR-based assays peaked on day 8 of fever, which demonstrated the detection rate to be between $31 \%$ and $44 \%$. All DNA-based assays (including isolation) markedly dropped to approximately $20 \%$ by day 10 of fever [14]. This reported drop in the PCR detection of O. tsutsugamushi in the later stages of scrub typhus fever may explain the absence of $O$. tsutsugamushi DNA in the majority of patients sampled in this study with suspected scrub typhus, while the PCRpositive cases may reflect the potential sampling of individuals during the earlier stages of the clinical presentation of this disease. These results nevertheless confirm that, by broad-range Rickettsiales $16 \mathrm{~S}$ rRNA PCR, O. tsutsugamushi DNA can be readily detected in blood samples of Nepalese patients, providing the potential for confirmatory testing if the assays are available for testing.

For the molecular characterization of these Nepalese O. tsutsugamushi strains, partial O. tsutsugamushi sequences were amplified from the $47 \mathrm{kDa}$ and $56 \mathrm{kDa}$ genes. The $56 \mathrm{kDa}$ type-specific antigen gene consists of unique and cross-reacting epitopes of Orientia, contributing to the serological diversity of this species [4]. As already mentioned, sequence analysis of the $56 \mathrm{kDa}$ type-specific antigen is recognized as a valuable and representative method for the genotyping of $O$. tsutsugamushi strains [15,16]. The $47 \mathrm{kDa}$ protein is more conserved than the $56 \mathrm{kDa}$ protein, but retains sufficient diversity to support genetic discrimination [6]. Our analysis of the partial $56 \mathrm{kDa}$ and $47 \mathrm{kDa}$ gene sequences from the Nepalese strains showed that, with the potential exception of Nepal_1, the strains are phylogenetically distinct from those reported in Guangdong, China [17], Thailand [18], and other regions in Asia [19]. For Nepal_1, 47kDa phylogenetic analysis placed this strain amidst the other Nepalese strains, in a subclade with another nearly identical Nepalese O. tsutsugamushi $47 \mathrm{kDa}$ sequence (98.7\% to Nepal_2; Figure 1). The $56 \mathrm{kDa}$ sequence analysis, however, placed Nepal_1 as a distinct lineage, clustering with a major clade of non-Nepalese O. tsutsugamushi strains (Figure 2). A potential explanation for the phylogenetic disparity for Nepal_1 may have to do with observations that the $56 \mathrm{kDa}$ encoding gene is recombinogenic, with in silico predictions that this O. tsutsugamushi gene regularly undergoes recombination events [20] as a part of the broader homologous recombination reported for this pathogen [21]. If this is the correct explanation, it would also imply that there is some level of geographic overlap in hosts that would support the recombination of phylogenetically distinct strains from Nepal and O. tsutsugamushi strains from elsewhere in Asia. 


\section{Materials and Methods}

\subsection{Study Population}

A cross-sectional descriptive study was conducted among patients with acute febrile illness with suspected scrub typhus from the Chitwan district in central Nepal for a duration of eight months, between April 2018 and November 2018. Cases of acute fever without localizing features that had more than four days duration were included in the study, following the exclusion of confirmed cases of other febrile illnesses including malaria, dengue, leptospirosis, and enteric diseases.

Blood samples were collected from the patients suspected of scrub typhus presenting with acute febrile illness in EDTA blood tubes. The buffy coat was separated and stored at $-80{ }^{\circ} \mathrm{C}$. During the time of admission, a structured questionnaire was completed to gain data, such as demographic variables, to analyze the disease epidemiology.

\subsection{Rickettsia PCR Screening and Sequencing}

DNA was extracted from the buffy coat using a HiYield ${ }^{\mathrm{TM}}$ DNA Mini Kit (YGB 300, Real Genomics, Taipei, Taiwan), and tested using rickettsial qPCR procedures established at the Australian Rickettsial Reference Laboratory (ARRL, Geelong, Australia) [22]. Briefly, the qPCR assay targeted the $O$. tsutsugamushi $16 \mathrm{~S}$ rRNA gene using primers $16 \mathrm{~S}$ rRNA-F (5'CTTATTTGCCAGCGGGTAATGC-3') and 16S rRNA-R (5'-GGGCCATGATGACTTGACCTC$3^{\prime}$ ), and the probe $16 \mathrm{~S}$ rRNA-Probe (5'-FAM-CCCACCTTCCTCCGGCTTAGCACCH-BQ10$\left.3^{\prime}\right)$. Each reaction contained $200 \mathrm{nM}$ of each primer and probe, $2 \times$ Platinum qPCR Super Mix-UDG Mastermix (Invitrogen, Melbourne, Australia), $5 \mathrm{mM} \mathrm{MgCl} 2$, and a DNA template to a total reaction volume of $25 \mu \mathrm{L}$. The reactions were performed and analyzed using the RotorGene 3000 (Corbett Research, Sydney, Australia) with positive and negative controls. The cycling parameters included an initial holding temperature of $50{ }^{\circ} \mathrm{C}$ for $3 \mathrm{~min}$, followed by $95^{\circ} \mathrm{C}$ for $5 \mathrm{~min}$ and 65 cycles of $95^{\circ} \mathrm{C}$ for $20 \mathrm{~s}$ and $60^{\circ} \mathrm{C}$ for $40 \mathrm{~s}$. Any samples with $\mathrm{Ct}$ values of $<35$ were deemed positive. Ct values between 35 and 40 were considered equivocal and were repeated for confirmatory testing. Ct values $<40$ were considered negative.

For rickettsial PCR-positive specimens, a conventional PCR targeting the $56 \mathrm{kDa}$ typespecific gene was performed using the forward (5'-TACATTAGCTGCAGGTATGACA- $\left.3^{\prime}\right)$ and reverse primers (5'-CCAGCATAATTCTTTAACCAAG-3') (Invitrogen, Melbourne, Australia), as previously described [23]. Additional sequence information for each rickettsial-positive specimen was obtained by PCR amplification of the $47 \mathrm{kDa}$ HtrA sequences using primers targeting members of the genus Orientia, as previously described [6]. Positive (Orientia tsutsugamushi Karp and Kato strains) and negative $\left(\mathrm{dH}_{2} \mathrm{O}\right)$ controls were included with every run for both PCRs.

Amplicon sequencing of the $56 \mathrm{kDa}$ and $47 \mathrm{kDa}$ PCR products was performed at Macrogen (Seoul, Korea). Sequences obtained were compared with sequences available by BlastN analysis (http://blast.ncbi.nlm.nih.gov accessed on 15 February 2021). The relationship of the Nepalese phylogenetic analysis was performed by the construction of the phylogenetic trees using the amplified $56 \mathrm{kDa}$ and $47 \mathrm{kDa}$ partial gene sequences separately. A phylogenetic tree was constructed from O. tsutsugamushi strains obtained from Genbank using the neighbor-joining method [24], as presented in MEGA 7 [25].

\section{Conclusions}

This study provides concrete molecular evidence for the presence of $O$. tsutsugamushi as the causative agent of scrub typhus in Nepal. This demonstration may help in the implementation of rapid diagnostics and early treatment of this disease in this region. Conventional PCR and the subsequent sequencing of the $56 \mathrm{kDa}$ and $47 \mathrm{kDa}$ antigens of this pathogen reveal that Nepalese strains are largely genetically distinct from those reported elsewhere in Asia, although we detected at least one strain that may share evolutionary ancestry with non-Nepalese $O$. tsutsugamushi strains through a process of homologous recombination. Knowledge of the circulating O. tsutsugamushi antigenic diversity of Nepalese 
strains may be important in the development of future diagnostic tests and vaccines that support efforts to control this important neglected tropical disease in this country.

Author Contributions: Conceptualization-R.G., K.P. and J.B.S.; Data curation-R.G. and K.P.; Formal analysis-M.T.; Funding acquisition-S.G. and J.S.; Investigation-R.G., M.T., J.S. and J.B.S.; Methodology-K.P. and J.B.S.; Project administration-K.P., M.T. and S.G.; Resources—S.G.; Supervision-J.B.S.; Visualization-J.S.; Writing—original draft, R.G. and M.T.; Writing—review and editing, S.G. and J.S. All authors have read and agreed to the published version of the manuscript.

Funding: This work was partially supported by University Grant Commission (Award No.: PhD 073/74/HS-01) and the Australian Rickettsial Reference Laboratory.

Institutional Review Board Statement: This study was approved by the ethics review board of the Institute of Medicine, Tribhuvan University, Kathmandu, Nepal (331/073/074).

Informed Consent Statement: Written informed consent was obtained from each patient prior to their enrolment in this study to publish the results of this work. Parents/guardians gave consent for child participants.

Data Availability Statement: The data presented in this study are openly available in GeneBank (for accession numbers see above).

Acknowledgments: We would like to acknowledge the faculty members from the Department of Microbiology, Maharajgunj Medical Campus, Institute of Medicine and all staff of Australian Rickettsial Reference Laboratory, Geelong, Victoria, Australia. We thank Adam Polkinghorne, Major Mitchell Consulting, for assistance in preparation of this manuscript.

Conflicts of Interest: The authors declare no conflict of interest.

\section{References}

1. Luce-Fedrow, A.; Lehman, M.L.; Kelly, D.J.; Mullins, K.; Maina, A.N.; Stewart, R.L.; Ge, H.; John, H.S.; Jiang, J.; Richards, A.L. A review of scrub typhus (Orientia tsutsugamushi and related organisms): Then, now, and tomorrow. Trop. Med. Infect. Dis. 2018, 3, 8. [CrossRef]

2. La Scola, B.; Raoult, D. Laboratory diagnosis of rickettsioses: Current approaches to diagnosis of old and new rickettsial diseases. J. Clin. Microbiol. 1997, 35, 2715-2727. [CrossRef] [PubMed]

3. Jiang, J.; Chan, T.C.; Temenak, J.J.; Dasch, G.A.; Ching, W.M.; Richards, A.L. Development of a quantitative real-time polymerase chain reaction assay specific for Orientia tsutsugamushi. Am. J. Trop. Med. Hyg. 2004, 70, 351-356. [CrossRef]

4. Ohashi, N.; Nashimoto, H.; Ikeda, H.; Tamura, A. Diversity of immunodominant 56-kDa type-specific antigen (TSA) of Rickettsia tsutsugamushi. Sequence and comparative analyses of the genes encoding TSA homologues from four antigenic variants. J. Biol. Chem. 1992, 267, 12728-12735. [CrossRef]

5. Ni, Y.S.; Chan, T.C.; Chao, C.C.; Richards, A.L.; Dasch, G.A.; Ching, W.M. Protection against scrub typhus by a plasmid vaccine encoding the 56-KD outer membrane protein antigen gene. Am. J. Trop. Med. Hyg. 2005, 73, 936-941. [CrossRef] [PubMed]

6. Jiang, J.; Paris, D.H.; Blacksell, S.D.; Aukkanit, N.; Newton, P.N.; Phetsouvanh, R.; Izzard, L.; Stenos, J.; Graves, S.R.; Day, N.P.; et al. Diversity of the 47-kD HtrA nucleic acid and translated amino acid sequences from 17 recent human isolates of Orientia. Vector Borne Zoonotic Dis. 2013, 13, 367-375. [CrossRef] [PubMed]

7. Kelly, D.J.; Fuerst, P.A.; Ching, W.M.; Richards, A.L. Scrub typhus: The geographic distribution of phenotypic and genotypic variants of Orientia tsutsugamushi. Clin. Infect. Dis. 2009, 48, S203-S230. [CrossRef]

8. Fournier, P.E.; Siritantikorn, S.; Rolain, J.M.; Suputtamongkol, Y.; Hoontrakul, S.; Charoenwat, S.; Losuwanaluk, K.; Parola, P.; Raoult, D. Detection of new genotypes of Orientia tsutsugamushi infecting humans in Thailand. Clin. Microbiol. Infect. 2008, 14, 168-173. [CrossRef]

9. $\quad$ Blacksell, S.D.; Luksameetanasan, R.; Kalambaheti, T.; Aukkanit, N.; Paris, D.H.; McGready, R.; Nosten, F.; Peacock, S.J.; Day, N.P. Genetic typing of the 56-kDa type-specific antigen gene of contemporary Orientia tsutsugamushi isolates causing human scrub typhus at two sites in north-eastern and western Thailand. FEMS Immunol. Med. Microbiol. 2008, 52, 335-342. [CrossRef]

10. Upadhyaya, B.P.; Shakya, G.; Adhikari, S.; Rijal, N.; Acharya, J.; Maharjan, L.; Marasini, B.R. Scrub typhus: An emergent neglected tropical disease in Nepal. J. Nepal. Health Res. Counc. 2016, 14, 122-127.

11. Gautam, R.; Parajuli, K.; Sherchand, J.B. Epidemiology, risk factors and seasonal variation of scrub typhus fever in Central Nepal. Trop. Med. Infect. Dis. 2019, 4, 27. [CrossRef]

12. Karki, K. Descriptive Epidemiology of Scrub Typhus in Nepal; Nepal Health Research Council: Kathmandu, Nepal, 2017.

13. Acharya, K.P.; Adhikari, N.; Tariq, M. Scrub typhys: A serious public health issue in Nepal. Clin. Epidemiol. Glob. Health 2020, 8 , 815-817. [CrossRef] 
14. Paris, D.H.; Blacksell, S.D.; Nawtaisong, P.; Jenjaroen, K.; Teeraratkul, A.; Chierakul, W.; Wuthiekanun, V.; Kantipong, P.; Day, N.P. Diagnostic accuracy of a loop-mediated isothermal PCR assay for detection of Orientia tsutsugamushi during acute scrub typhus infection. PLoS Negl. Trop. Dis. 2011, 5, e1307. [CrossRef]

15. Long, J.; Wei, Y.; Tao, X.; He, P.; Xu, J.; Wu, X.; Zhu, W.; Chen, K.; Yang, Z. Representative genotyping, recombination and evolutionary dynamics analysis of TSA56 gene segment of Orientia tsutsugamushi. Front. Cell. Infect. Microbiol. 2020, 10, 383. [CrossRef]

16. Kelly, D.J.; Fuerst, P.A.; Richards, A.L. Origins, importance and genetic stability of the prototype strains Gilliam, Karp and Kato of Orientia tsutsugamushi. Trop. Med. Infect. Dis. 2019, 4. [CrossRef]

17. De, W.; Jing, K.; Huan, Z.; Qiong, Z.H.; Monagin, C.; Min, Z.J.; Ping, H.; Wen, K.C.; Yan, L.J. Scrub typhus, a disease with increasing threat in Guangdong, China. PLoS ONE 2015, 10, e0113968. [CrossRef]

18. Ruang-Areerate, T.; Jeamwattanalert, P.; Rodkvamtook, W.; Richards, A.L.; Sunyakumthorn, P.; Gaywee, J. Genotype diversity and distribution of Orientia tsutsugamushi causing scrub typhus in Thailand. J. Clin. Microbiol. 2011, 49, 2584-2589. [CrossRef]

19. Takhampunya, R.; Tippayachai, B.; Promsathaporn, S.; Leepitakrat, S.; Monkanna, T.; Schuster, A.L.; Melendrez, M.C.; Paris, D.H.; Richards, A.L.; Richardson, J.H. Characterization based on the 56-Kda type-specific antigen gene of Orientia tsutsugamushi genotypes isolated from Leptotrombidium mites and the rodent host post-infection. Am. J. Trop. Med. Hyg. 2014, 90, 139-146. [CrossRef]

20. Kim, G.; Ha, N.Y.; Min, C.K.; Kim, H.I.; Yen, N.T.; Lee, K.H.; Oh, I.; Kang, J.S.; Choi, M.S.; Kim, I.S.; et al. Diversification of Orientia tsutsugamushi genotypes by intragenic recombination and their potential expansion in endemic areas. PLoS Negl. Trop. Dis. 2017, 11, e0005408. [CrossRef]

21. Sonthayanon, P.; Peacock, S.J.; Chierakul, W.; Wuthiekanun, V.; Blacksell, S.D.; Holden, M.T.; Bentley, S.D.; Feil, E.J.; Day, N.P. High rates of homologous recombination in the mite endosymbiont and opportunistic human pathogen Orientia tsutsugamushi. PLoS Negl. Trop. Dis. 2010, 4, e752. [CrossRef]

22. Stenos, J.; Graves, S.; Izzard, L. Rickettsia. In PCR for Clinical Microbiology-An Australian and International Perspective; Schuller, M., Sloots, T.P., James, G.S., Halliday, C.L., Carter-Wientjes, C., Eds.; Springer: Dordrecht, The Netherlands, 2010.

23. Unsworth, N.B.; Stenos, J.; Faa, A.G.; Graves, S.R. Three rickettsioses, Darnley Island, Australia. Emerg. Infect. Dis. 2007, 13, 1105-1107. [CrossRef] [PubMed]

24. Saitou, N.; Nei, M. The neighbor-joining method: A new method for reconstructing phylogenetic trees. Mol. Biol. Evol. 1987, 4, 406-425. [CrossRef] [PubMed]

25. Kumar, S.; Stecher, G.; Tamura, K. MEGA7: Molecular evolutionary genetics analysis version 7.0 for bigger datasets. Mol. Biol. Evol. 2016, 33, 1870-1874. [CrossRef] [PubMed] 